\author{
Military Technical College \\ Kobry El-kobbah, \\ Cairo, Egypt
}

\author{
$5^{\text {th }}$ International Conference \\ on Electrical Engineering \\ ICEENG 2006
}

\title{
STUDY AND COMPARISON BETWEEN METHODS AND ALGORITHMS OF PULSE OXIMETER SIGNAL ANALYSIS
}

\author{
MOHAMED* A.M. and SERRY* S.B.
}

\begin{abstract}
Now a day Pulse Oximeter is a very important instrument in the surgical or intensive care units, as it's a very fast and real time indication to the blood oxygen saturation level, which gives an indication of the patient's status. Its theory of operation mainly depends on the phenomena of difference in the absorption of different wavelengths by body tissues and blood constitutes. The raw Pulse Oximeter (plethysmography) signal undergoes a lot of processing to calculate the desired values of the Blood Oxygen saturation .Many methods are employed either in time domain analysis or through frequency domain analysis. Each Method has its own advantage and disadvantage. By combining all these methods into a hybrid algorithm of processing taking into consideration the limitations and the most important advantages in each method, it is applicable to have an accurate method of calculating the $\mathrm{SaO}_{2}$.
\end{abstract}

\section{KEYWORDS}

Pulse Oximeter ,FFT ,Moving Average Technique

\section{NOMENCLATURE}

$\begin{array}{lll}\mathrm{SpO}_{2} & \ldots & \text { Pulse Oximeter Oxygen Saturation Reading } \\ \mathrm{SaO}_{2} & \ldots & \text { Arterial Oxygen Saturation } \\ \mathrm{Hb} & \ldots & \text { Hemoglobin Concentration } \\ \mathrm{FFT} & \ldots & \text { Fast Fourier Transform } \\ \mathrm{DFT} & \ldots & \text { Discrete Fourier Transform } \\ \mathrm{DWT} & \ldots & \text { Discrete Wavelet Transform } \\ \mathrm{AC} & \ldots & \text { Received Signal AC Component } \\ \mathrm{DC} & \ldots & \text { Received Signal DC Component } \\ \mathrm{R} & \ldots & \text { Ratio of Ratios (Ratio between Red AC/DC to IR AC/DC) } \\ \mathrm{r}_{\mathrm{a}} & \ldots & \text { Optical Density ratio } \\ \mathrm{r}_{\mathrm{a}} & \ldots & \text { Arterial optical density ratio } \\ \mathrm{r} & \ldots & \text { Venous optical density ratio } \\ \text { ANC } & \ldots & \text { Adaptive Noise Canceller }\end{array}$




\section{INTRODUCTION}

A blood-oxygen saturation reading indicates the percentage of hemoglobin molecules in the arterial blood which are saturated with oxygen. The reading may be referred to as $\mathrm{SaO}_{2}$. This can be done by using an optical Vivo measurement method which is the Pulse Oximeter .Arterial oxygen saturation $\left(\mathrm{SaO}_{2}\right)$ simply represents the grams of available hemoglobin concentration $[\mathrm{Hb}]$ which are carrying oxygen. The main sources of interference and noise for the Pulse Oximeter signal are Light Interference as sometimes external light sources may cause inaccurate readings. Bright lights, including infrared heat lamps, may interfere and cause a higher $\mathrm{SpO} 2$ and pulse rate than is really occurring. Movement Artifacts where any movement of the patient (seizures, shivering etc...), sensor, stretcher, or the transporting vehicle, unusually strong movement may cause movement artifact in the pulse [1]. Analysis can be done either through time domain by using moving average technique or through frequency domain FFT or DFT by using the discrete wavelet transform (DWT) to calculate the arterial oxygen saturation (SaO2) [2]. Our work here propose a new hybrid method combining all of those methods in a way to overcome the main disadvantage of each method and overcoming all the sources of noise and interference and having an accurate reading of SaO2.

\section{DESCRIPTION OF VARIOUS ANALYSIS METHODS}

All methods and algorithms end with same empirical formula which calculate a ratio $\mathrm{R}$ which depends on the $\mathrm{AC}$ and $\mathrm{DC}$ component of the transmitted signal through (reflected signal from) the body tissues [1]

$$
R=\frac{\left(\frac{A C_{r e d}}{D C_{r e d}}\right)}{\left(\frac{A C_{I r}}{D C_{I r}}\right)}
$$

Where this ratio is used to calculate the $\mathrm{SpO}_{2}$ value using another empirical formula [1]

$$
\mathrm{SpO}_{2}=-25 R+110
$$

But the Analysis and the processing of different methods to get the most accurate values of the $A C$ and DC components used in the calculations are different.

In Time domain analysis it is accomplished by the comparison between the amplitude of AC and DC component of both the Red and IR signals detected by the Photodiode after propagation inside the tissues, where the $\mathrm{R}$ values were computed by a commercial vendor's algorithm approximately once every second. This algorithm analyzed the time series waveform via analog filtering and weighted moving techniques, and approximated the $A C$ and $D C$ values strictly in the time domain. Next, the time series $\mathrm{R}$ values were averaged over the 8.53 second data collection period [3]. 
In frequency domain analysis, the Fast Fourier transformation of the time series waveform via a simple software program that compute the magnitude of the highest spectral peak representing the pulsatile, or $\mathrm{AC}$ component of the oximetery waveform (between $0.5-2.5 \mathrm{~Hz}$ ). In addition the magnitude of the DC offset component was found to be as well $(\mathrm{OHz})$. After which the $\mathrm{R}$ is calculated on from the red and IR AC and DC spectral peaks, based on the formula [3].

\section{DISCUSSION}

The desired signal has been acquired using a finger probe (Pulse Oximeter Probes can be in different types: Finger probe, ear lope probe ...etc). The detected physiological signal is generally composed of both desired signal (S) and undesired signal $(\mathrm{N})$ or noise portions. The Signal and the Noise are seen in Fig.1 for both Time Domain and Frequency Domain. To remove the effects of the undesired signal, some knowledge of the noise characteristics, or equivalently its noise reference ( $\left.\mathrm{N}^{\prime}\right)$, must be known. The Raw signal consists all of: a) a DC offset component, b) a respiratory component, c) an AC component, d) an electrical interference component, and e) the signal itself. Three main types of interference are of particular interest during the signal processing phase: electrical noise, motion artifacts, and respiratory variation.
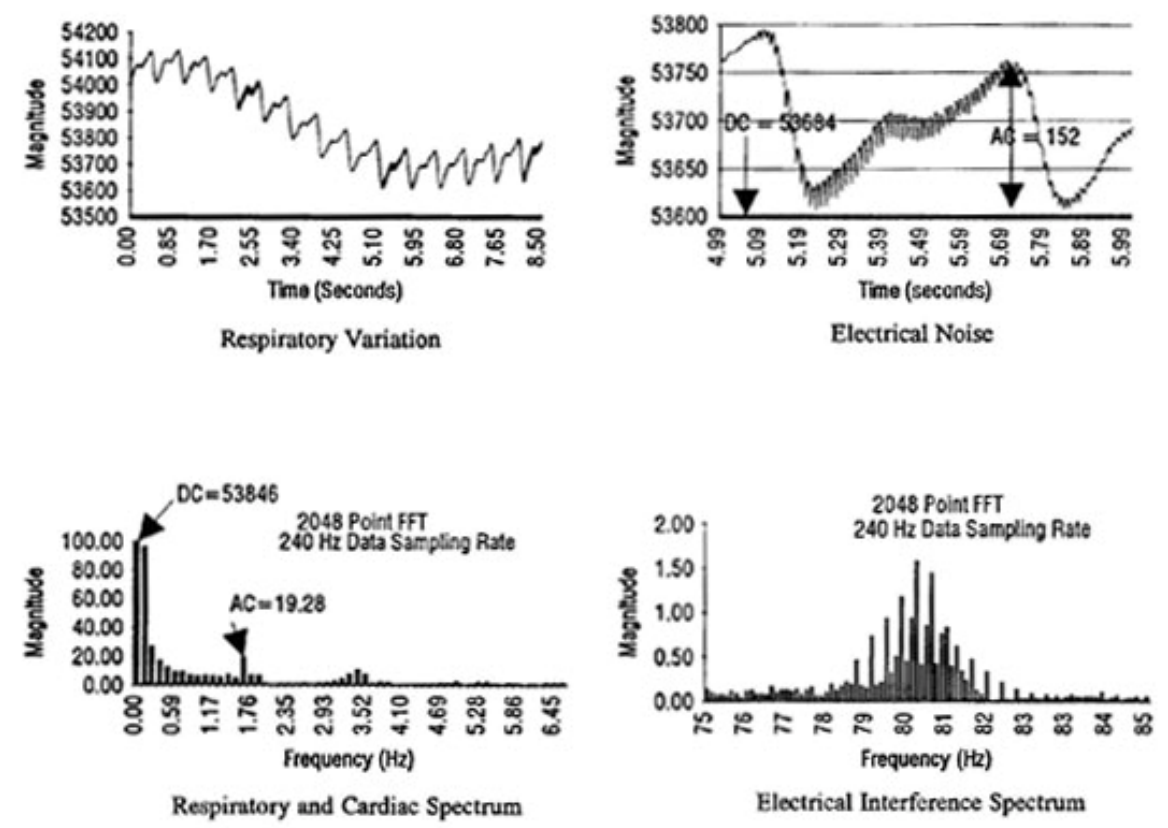

Fig.1. Signal and Noise in both Time Domain and Frequency Domain

By studying the frequencies of these main three types of interference, it was found that a) the respiratory variation is at $(0.08-0.5 \mathrm{~Hz}), \mathrm{b})$ Motion artifacts component is at $(0.5-2.5 \mathrm{~Hz})$, and c) the electrical noise are at $(60-80 \mathrm{~Hz})$. 
For time series analysis: high frequency electrical noise is removed via a preprocessing low pass analog filter while in spectral analysis, Low frequency respiratory variation is removed via a pre-processing high pass analog filter, and for the motion artifact it remain a difficult problem as it lies in the same spectrum of interest.

For Spectral analysis: high frequency electrical noise can be easily removed from the spectrum of interest, Low frequency respiratory variation can potentially be calculated the respiratory rate based on the highest spike found within the respiratory variation spectrum band, and for the motion artifact can be separated from the AC Oximeter signal based on its spectrum, even it can be considered a problem if its component is in the same frequency of the signal in interest.

\section{DESCRIPTION OF THE NEW HYBRID METHOD}

By combining all the stated methods together into a new Hybrid algorithm in the following steps: 1) A band-pass analog filter to reject both the DC offset component and the electrical noise taking into account the following steps, as shown in Fig. 2 .



Fig.2. Signal and Noise after being introduced to a Band-Pass Filter

2) Using software to convert the signal from time domain into frequency domain by FFT .3) Using an Adaptive filter which derives its name from its ability to change its filtering characteristics in response to changing in-band noise, having the advantage that it is a digital filter contained within the memory of the microprocessor as it is a software processing with no additional hardware introduced to the Pulse Oximeter system and can be rapidly changed, which allows its weight coefficients (Noise Reference) to be changed in real time. Thus, the filter can be tuned "on the fly". Where these coefficients determine whether the frequency components of an input signal should be cancelled. It depends on generating a Noise reference (N') depending on the estimation of the optical density ratio ( $r$ ) and for each selected value of the optical density ratio [4]. Where the detected physiologic signals in response to both red $\left(\mathrm{I}_{\mathrm{rd}}\right)$ and infrared $\left(\mathrm{I}_{\mathrm{ir}}\right)$ light consist of desired signal portions $\left(\mathrm{S}_{\mathrm{rd}}\right.$, $\mathrm{S}_{\mathrm{ir}}$ ) as well as undesired signal portions $\left(\mathrm{N}_{\mathrm{rd}}, \mathrm{N}_{\mathrm{ir}}\right)$. It is commonly understood in pulse oximetry that the desired signal portions are proportional to one another through the arterial optical density ratio $\left(r_{a}\right)$. This suggests that one should simply subtract the product of the arterial optical density ratio and the physiologic signal due to infrared light from the physiologic signal due to red light. The resultant is a reference signal 
that contains only noise portions. This is the noise reference signal $\left(\mathrm{N}^{\prime}\right)$. As seen in Fig.3.

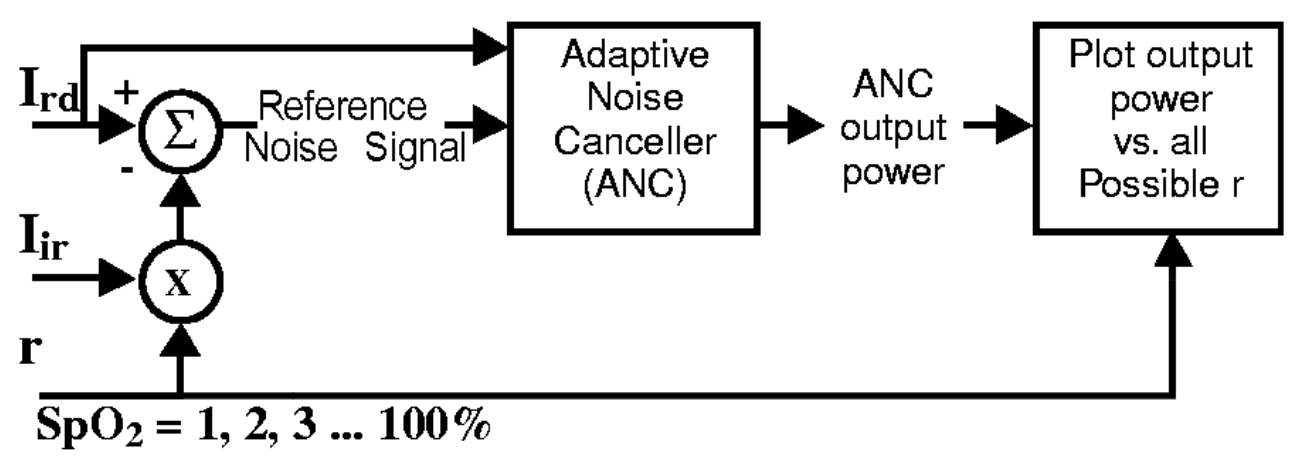

Fig.3. The Adaptive Digital Filter

Since [4]:

$$
\begin{aligned}
& I_{r d}=S_{r d}+N_{r d} \\
& I_{i r}=S_{i r}+N_{i r} \\
& r_{a}=\frac{S_{r d}}{S_{i r}}>S_{r d}=r_{a} \cdot S_{i r}
\end{aligned}
$$

Then:

$$
I_{r d}-\left[I_{i r} \cdot r_{a}\right]=\left[S_{r d}+N_{r d}\right]-\left[S_{i r} r_{a}+{ }_{i r} r_{a}\right]
$$

Substituting $S_{i r} r_{a}$ for $S_{r d}$, we get:

$$
\begin{aligned}
I_{r d}-\left[I_{i r} . r_{a}\right] & =\left[S_{i r} r_{a}+N_{r d}\right]-\left[S_{i r} r_{a}+N_{i r} r_{a}\right] \\
& =N_{r d}-N_{i r} r_{a} \\
& =N^{\prime}
\end{aligned}
$$

Where $N^{\prime}$ is the noise reference. The corresponding reference signal is calculated and subsequently processed through an adaptive noise canceller (ANC). As Shown in fig.4. Where the peaks of the resultant spectrum represent the values of the $R$ value which is used to calculate the SpO2 from equation (2). 




Fig.4. The Adaptive Filter and ANC spectral output

\section{CONCLUSION}

The use of the advantage of both methods of signal analysis of the Pulse Oximeter signal can be combined in a Hybrid Analysis method which can increase the accuracy and the efficiency of the Pulse Oximeter signal without introducing any new hardware to the Pulse Oximeter system. Where a Sweep of all optical density ratios that correspond to oxygen saturations of $1 \%$ to $100 \%$. Computing the reference signal for each optical density ratio. Measuring the output power of the adaptive noise canceller for each reference signal. Then identifying the appropriate peak that corresponds to the arterial oxygen saturation (largest SpO2 value).

\section{REFERENCES}

[1] J.G.Webster, "Design of Pulse Oximeters" (1997).

[2] J.M.Kim, S.H.Kim, D.J.Lee, H.S. Lim, Applied Spectroscopy, November 2001, vol. 55, no. 11, pp. 1435-1447(13), Ingenta. "Signal processing using Fourier and wavelet transform for pulse oximetery", (2001).

[3] E.S.John, A.Stephan, "Pulse Oximetery through Spectral Analysis" , IEEE (1993) http://203.162.7.79/ieee/pdf/disk 51/691/6333 /227 229 Pulse \%20oximetry \%20through \%20sp.pdf,

[4] Masimo Corporation, Technical Bulletin \#1 http://www.masimo.com/pdf/technology/LAB1035M.pdf 